\title{
Influência da competição intra-específica entre fêmeas e da ausência de hospedeiro no parasitismo de Telenomus remus Nixon (Hymenoptera, Scelionidae) sobre ovos de Spodoptera frugiperda (J. E. Smith) (Lepidoptera, Noctuidae)
}

\author{
Tatiana R. Carneiro ${ }^{1}$, Odair A. Fernandes ${ }^{1,2} \&{\text { Ivan } \text { Cruz }^{3}}^{2}$
}

${ }^{1}$ Departamento de Fitossanidade, Universidade Estadual Paulista, Via de Acesso Prof. Paulo Donato Castellane, km 5 14884-900 Jaboticabal-SP, Brasil.tatianacarneiro@hotmail.com; oafernandes@fcav.unesp.br

${ }^{2}$ Pesquisador do $\mathrm{CNPq} /$ autor correspondente.

${ }^{3}$ Embrapa Milho e Sorgo, Caixa Postal 151, 35701-970 Sete Lagoas-MG, Brasil. ivancruz@cnpms.embrapa.br

\begin{abstract}
Influence of females intraspecific competition and lack of host on Telenomus remus Nixon (Hymenoptera, Scelionidae) parasitism on Spodoptera frugiperda (J. E. Smith) (Lepidoptera, Noctuidae) eggs. Intraspecific competition and lack of host can affect the success of biological control programs. Thus, this work aimed at evaluating the effect of different densities of females (1, 3, 6, and 9 females/100 eggs) and the absence of host in the parasitism of Telenomus remus Nixon (Hymenoptera, Scelionidae) on Spodoptera frugiperda (J.E. Smith) (Lepidoptera, Noctuidae) eggs. It was observed an increase in egg parasitism of $S$. frugiperda by T. remus beyond a density of 3 females/ 100 eggs under laboratory (18\%) and field (32\%) conditions, although the parasitism caused by densities greater than 1 female/100 eggs was not significantly different. However, this did not cause superparasitism. On the other hand, the number of females in the progeny was lower (39\%) as density of females increased, but it was not observed in field conditions. Even after nine days of host absence there was no significant reduction in the parasitism which was ca. 95 eggs/female/day. However, a decrease in the number of parasitized eggs $(40 \%)$ could be observed on the 10th day. Therefore, the release of three females per 100-egg mass is adequate for high levels of parasitism.
\end{abstract}

KEYWORDS. Biological control; egg parasitoid; fall armyworm; maize.

RESUMO. Influência da competição intra-específica entre fêmeas e da ausência de hospedeiro no parasitismo de Telenomus remus Nixon (Hymenoptera, Scelionidae) sobre ovos de Spodoptera frugiperda (J. E. Smith) (Lepidoptera, Noctuidae). A competição intraespecífica e a ausência de hospedeiros podem comprometer o sucesso de programas de controle biológico. Assim, este trabalho objetivou avaliar o efeito de diferentes densidades de fêmeas (1, 3, 6 e 9 fêmeas/ 100 ovos) e da ausência de hospedeiro no parasitismo de Telenomus remus Nixon (Hymenoptera, Scelionidae) em ovos de Spodoptera frugiperda (J. E. Smith) (Lepidoptera, Noctuidae). Observou-se aumento no parasitismo de ovos de $S$. frugiperda por T. remus a partir da densidade 3 fêmeas/ 100 ovos tanto em condições de laboratório (18\%) quanto em campo (32\%), embora o parasitismo causado por densidades maiores que 1 fêmea/100 ovos não foi significativamente diferente. Todavia, isso não causou superparasitismo. Por outro lado, o número de fêmeas produzidas na progênie diminuiu (39\%) com a maior densidade de fêmeas sob condições de laboratório. Contudo, não verificou-se diferença significativa sob condições de campo. Mesmo após 9 dias de ausência de hospedeiro não houve redução nos índices de parasitismo, permanecendo em torno de 95 ovos parasitados/fêmea/dia. Todavia, no décimo dia pode-se observar redução (40\%) no número médio de ovos parasitados. Desse modo, a liberação de três fêmeas por 100 ovos é adequada para se obter elevados níveis de parasitismo.

PALAVRAS-CHAVE. Controle biológico; lagarta-do-cartucho; milho; parasitóide de ovos.

Na concepção de manejo integrado, a meta não se trata de exterminar a praga, mas de manter sua população regulada, reduzindo-a a um limite compatível com a produção econômica da cultura e a conseqüente manutenção da qualidade ambiental (Cruz 1995). Logo, deve-se sempre ter atenção aos fatores de supressão e regulação de populações, avaliando-se as densidades populacionais da praga e de seus inimigos naturais.

Uma das fases fundamentais para que um programa de manejo integrado que utiliza parasitóides obtenha sucesso é o estabelecimento inicial do agente de controle biológico, atuando sobre a praga-alvo (Parra et al. 2002). Entretanto, para que isso ocorra faz-se necessário verificar a disponibilidade do hospedeiro na área e sua flutuação populacional, a fim de evitar que o inimigo natural passe por períodos de privação e acabe por migrar ou não se estabelecer (Hougardy et al. 2005). Poucos estudos foram desenvolvidos com o intuito de verificar o efeito da privação de hospedeiros sobre inimigos naturais, mesmo entendendo-se que tal fator pode limitar o sucesso de programas de controle biológico.

Durante a fase adulta, ao passar por períodos sem a disponibilidade de hospedeiros, fêmeas de algumas espécies de parasitóides de ovos podem ajustar a produção de ovos por determinado período, até encontrarem o inseto apropriado para sua oviposição (Hougardy et al. 2005). É comum verificar 
a absorção dos oócitos quando o alimento e/ou o hospedeiro não está disponível (Garcia 1991), ou ainda as fêmeas podem reter os ovos e compensar o período de ausência de hospedeiro com uma extensão da vida reprodutiva (Bai \& Smith 1993; Zera \& Harshman 2001).

Após estarem estabelecidos, os parasitóides precisam manter sua população, sem eliminar o hospedeiro. Para isso possuem estratégias reprodutivas, como a manipulação da razão sexual, quando encontram-se em menores ou maiores densidades de fêmeas (Waage \& Hassell 1982). A competição intra-específica entre fêmeas, especialmente em Scelionidae, é capaz de ocasionar mudanças na razão sexual da prole, principalmente em criações em laboratório (Waage 1982), inclusive de Telenomus remus Nixon (Hymenoptera, Scelionidae) (Welzen \& Waage 1987).

Tais fatos influenciam diretamente na atuação do agente de controle biológico, pois após longos períodos de ausência de hospedeiro, parasitóides costumam apresentar superparasitismo (Collins \& Dixon 1986), o que pode comprometer a qualidade da prole (Garcia 1991; Cave 2000). Além disso, o tempo que a fêmea leva para encontrar seu hospedeiro também tem sido relacionado como um fator que interfere em sua taxa de parasitismo (Berti \& Marcano 1991; Oliveira et al. 2000).

Sendo assim, visto que um dos fatores limitantes à expansão do uso de agentes de controle biológico na cultura do milho diz respeito à otimização das técnicas de criação de inimigos naturais (Cruz 2000) e percebendo-se a necessidade de estudos relacionados às características biológicas e à criação massal de T. remus, este trabalho objetivou avaliar o efeito da ausência de hospedeiros e da competição intra-específica entre fêmeas sobre a biologia deste inimigo natural.

\section{MATERIALE MÉTODOS}

Efeito da competição intra-específica entre fêmeas.

Testes em laboratório. O experimento foi realizado com quatro tratamentos, que consistiram em diferentes densidades de fêmeas de $T$. remus (1, 3, 6 e 9), expostas a posturas de Spodoptera frugiperda (J. E. Smith) (Lepidoptera, Noctuidae) contendo 100 ovos. Para a contagem dos ovos utilizou-se o método proposto por Beserra \& Parra (2005). Cada um dos tratamentos foi composto por 10 repetições.

Em tubos de vidro ( $8 \mathrm{~cm}$ de altura e $2 \mathrm{~cm}$ de diâmetro), foram individualizadas fêmeas do parasitóide $(\leq 24 \mathrm{~h})$, nas densidades propostas, introduzindo-se uma cartela contendo 100 ovos do hospedeiro em cada tubo. Foi permitida a oviposição por 24 h e após este período, as cartelas foram retiradas.

Para todas as fêmeas utilizadas disponibilizou-se como alimento pequenas gotas de mel, dispostas na parede interna dos tubos. Durante todo o experimento os tubos contendo fêmeas e cartelas com posturas de $S$. frugiperda foram mantidos em câmara climatizada $\left(25 \pm 1^{\circ} \mathrm{C}, 70 \pm 10 \%\right.$ UR e fotofase de $\left.12 \mathrm{~h}\right)$. As lagartas eclodidas foram contadas e retiradas dos tubos com o auxílio de um pincel, para que não danificassem os possíveis ovos parasitados. Os parasitóides emergidos dos ovos restantes foram, após a morte, contados e separados por sexo, com o auxílio de microscópio estereoscópico. Os parâmetros avaliados foram número de ovos parasitados, número de indivíduos emergidos/ovo, razão sexual e viabilidade.

Testes em campo. $\mathrm{O}$ experimento deu-se de maneira semelhante àquele conduzido em laboratório. No entanto, as fêmeas de $T$. remus $(\leq 24 \mathrm{~h})$, nas densidades propostas $(1,3,6$ e 9 fêmeas), foram liberadas em gaiolas confeccionadas em tecido "voile" (1,70 m de altura e $1 \mathrm{~m}$ de diâmetro). Foram adotadas cinco repetições. Em cada gaiola havia quatro plantas de milho com 20 dias após a emergência (V4 a V6) e uma cartela, afixada a uma das plantas, contendo 100 ovos do hospedeiro. As cartelas foram afixadas com auxílio de grampeador, na folha mais próxima ao cartucho. Foi permitida a oviposição por $48 \mathrm{~h}$, pois o tempo de busca dos parasitóides pode ser afetado pelo maior espaço físico nas gaiolas. Após este período as cartelas foram retiradas e avaliadas de modo semelhante ao realizado nos testes em laboratório.

Efeito da ausência de hospedeiro. Para determinar o efeito da ausência de hospedeiro sobre o parasitismo de T. remus por diferentes períodos de tempo $(1,2,3,4,5,6,7,8,9$ e 10 dias), 100 fêmeas do parasitóide, recém-emergidas $(\leq 24$ h) foram individualizadas em tubos de vidro $(8 \mathrm{~cm}$ de altura e 2 $\mathrm{cm}$ de diâmetro). Diariamente, durante 10 dias, foram separados 10 tubos contendo fêmeas, que eram então expostas a uma cartela contendo 100 ovos de $S$. frugiperda.

Foi permitida a cada fêmea a oviposição por $24 \mathrm{~h}$ e, após este período, as cartelas foram retiradas. No decorrer dos 10 dias de experimento as fêmeas de $T$. remus que aguardavam individualizadas para realizar postura foram mantidas com mel, disponibilizado em forma de pequenas gotas depositadas na parede do tubo.

Durante todo o experimento os tubos contendo fêmeas e cartelas com posturas de $S$. frugiperda foram mantidos em câmara climatizada regulada a $25 \pm 1^{\circ} \mathrm{C}, 70 \pm 10 \%$ UR e fotofase de $12 \mathrm{~h}$. As lagartas eclodidas foram contadas e retiradas dos tubos com o auxílio de um pincel, para que não danificassem os possíveis ovos parasitados. Os parasitóides emergidos dos ovos restantes foram, após a morte, contados e separados por sexo, sob microscópio estereoscópico. O delineamento utilizado foi inteiramente casualizado com 10 repetições.

Análise estatística. Tanto nos testes em laboratório como naqueles realizados no campo, foram avaliados o parasitismo, percentual de emergência de descendentes (viabilidade), razão sexual e número de descendentes emergidos por ovo. O delineamento utilizado foi inteiramente casualizado e as médias foram comparadas pelo Teste de Tukey a 5\% de probabilidade utilizando o procedimento PROC GLM (SAS Institute 2004).

\section{RESULTADOS EDISCUSSÃO}

Efeito da competição intra-específica entre fêmeas. Após a avaliação dos ovos parasitados, foi possível constatar aumento de $18 \%$ no parasitismo de ovos de S. frugiperda por T. remus a partir da densidade de 3 fêmeas/ 100 ovos (Tabela 
Tabela I. Parasitismo, número de indivíduos por ovo, razão sexual e viabilidade ( \pm EPM) de $T$. remus sobre $S$. frugiperda, mediante diferentes densidades de fêmeas do parasitóide, em condições de laboratório.

\begin{tabular}{ccccc}
\hline $\begin{array}{c}\text { Fêmeas/ } \\
100 \\
\text { ovos }\end{array}$ & $\begin{array}{c}\text { No. de ovos } \\
\text { parasitados }\end{array}$ & $\begin{array}{c}\text { No. de } \\
\text { indivíduos/ } \\
\text { ovo }\end{array}$ & $\begin{array}{c}\text { Razão } \\
\text { sexual }\end{array}$ & $\begin{array}{c}\text { Viabilidade } \\
(\%)\end{array}$ \\
\hline 1 & $84,8 \pm 2,8$ b & $1,0 \pm 0,00$ a & $0,70 \pm 0,09$ a & $99,6 \pm 0,04$ a \\
3 & $99,7 \pm 0,3$ a & $1,1 \pm 0,03$ a & $0,69 \pm 0,03$ a & $99,2 \pm 0,01$ a \\
6 & $99,9 \pm 0,1$ a & $1,1 \pm 0,03$ a & $0,60 \pm 0,06$ ab & $99,8 \pm 0,01 \mathrm{a}$ \\
9 & $100,0 \pm 0,0$ a & $1,1 \pm 0,02$ a & $0,43 \pm 0,05$ b & $99,7 \pm 0,02$ a \\
\hline
\end{tabular}

Médias seguidas da mesma letra, na coluna, não diferem significativamente, entre si, pelo teste de Tukey $(\mathrm{P} \leq 0,05)$.

I), em condições de laboratório, pois, uma única fêmea do parasitóide à temperatura de $25^{\circ} \mathrm{C}$ parasita, nas primeiras 24 horas, em média, 81,75 ovos (Bueno et al. 2008). Logo, o aumento nas densidades de fêmeas ocasionou o aumento do parasitismo.

O aumento do parasitismo também foi constatado sob condições de campo, porém foi maior (32\%) (Tabela II). Percebe-se que a partir da densidade de 3 fêmeas/ 100 ovos há um aumento do número de ovos parasitados, porém não há diferença significativa entre as densidades de 3, 6 e 9 fêmeas/ 100 ovos. Cabe ressaltar, entretanto, que mesmo com altas densidades de fêmeas/ovo, o parasitismo não foi prejudicado, pois fêmeas de $T$. remus toleram a presença de outras fêmeas ovipositando sobre a mesma massa de ovos (Schwartz \& Gerling 1974).

Apesar do menor número de ovos parasitados sob condições de campo, especialmente na densidade 1 fêmea/ 100 ovos, as taxas de parasitismo continuaram altas, expressando sempre médias superiores a $60 \%$ da massa de ovos parasitada (Tabela II).

Mesmo com o aumento na densidade de fêmeas, não se detectou mudanças significativas no número de indivíduos por ovo (Tabelas I e II). Isso decorre, pois quando fêmeas de T. remus encontram-se em altas densidades tendem a diminuir o número de ovos depositados/ fêmea (Welzen \& Waage 1987). Tal fato pode ser explicado, pois, após depositar os ovos, as fêmeas raspam o cório do ovo do hospedeiro com seu ovipositor (Schwartz \& Gerling 1974; Welzen \& Waage 1987) para evitar oviposição repetida, sendo tal comportamento comum nos insetos da família Scelionidae (Gauld \& Bolton 1988). Apesar da fêmea realizar este tipo de marcação nos ovos parasitados, Schwartz \& Gerling (1974) registraram que tal ação não é capaz de evitar o superparasitismo na primeira hora após a oviposição.

Acredita-se que as baixas taxas de superparasitismo encontradas na espécie devem-se à competição intraespecífica das larvas e/ou pela falta de nutrientes dentro do ovo do hospedeiro, que permitiriam a sobrevivência de apenas um indivíduo (Gerling 1972; Cave 2000). Porém, apesar da competição, não há prejuízos à viabilidade dos insetos, em condições de laboratório (Tabela I).

Sob condições de campo, as taxas de viabilidade de $T$. remus, em todas as densidades estudadas, mantiveram-se em torno dos 50\%, em média (Tabela II). Acredita-se que tal decréscimo nos valores referentes à viabilidade dos insetos, quando comparados àqueles testados em laboratório, devase aos fatores abióticos aos quais os ovos parasitados permaneceram expostos durante o período em que estiveram no campo. Isso decorre, pois mesmo nestas condições não constatou-se a presença de mais de um indivíduo por ovo (Tabela II).

Com relação ao parâmetro razão sexual, pode-se perceber neste ensaio que o número de fêmeas produzidas em laboratório diminuiu com a maior densidade de fêmeas, diferenciando-se significativamente dos demais tratamentos (Tabela I). Welzen \& Waage (1987), ao trabalharem com as densidades de 1,2 e 4 fêmeas de $T$. remus por postura do hospedeiro demonstraram também que na maior densidade houve uma maior tendência de produção de machos. Porém, os autores não mantiveram as fêmeas em conjunto, mas ofertaram massas de ovos previamente parasitadas por cada uma das fêmeas às outras separadamente. A proporção dos sexos na prole de T. remus é normalmente de 60 a $70 \%$ de fêmeas (Cave \& Acosta 1999), porém pode modificar-se devido a fatores abióticos, como a temperatura (Bueno et al. 2008), ou fatores bióticos como a densidade de fêmeas (Doutt 1959; Welzen \& Waage 1987).

Quando apenas uma fêmea de parasitóide coloniza um hospedeiro ou uma única porção de hospedeiros (como massas de ovos, por exemplo), existe a tendência de que a mesma produza apenas machos suficientes para fertilizar as fêmeas que depositou naquele(s) hospedeiro(s). No entanto, quando várias fêmeas colonizam um único hospedeiro ou uma única porção de hospedeiros, cada fêmea precisa produzir o número suficiente de machos para competir com os descendentes machos produzidos pelas outras fêmeas (Van Alphen \& Jervis 1996).

A maioria dos Scelionidae são solitários, mas costumam atacar hospedeiros que encontram-se em grupo (Gauld \& Bolton 1988) e dentre tais parasitóides T. remus destaca-se por sua preferência por ovos em massa (Vinson 1998). Contudo, diante da presença de outras fêmeas na mesma postura exibem mudanças na alocação sexual e, como resposta, produzem mais descendentes machos, mas apenas o fazem quando a proporção de ovos não parasitados por fêmea é muito pequena (Welzen \& Waage 1987).

Tabela II. Parasitismo, número de indivíduos por ovo, razão sexual e viabilidade ( \pm EPM) de $T$. remus sobre $S$. frugiperda, mediante diferentes densidades de fêmeas do parasitóide, em condições de campo.

\begin{tabular}{ccccc}
\hline $\begin{array}{c}\text { Fêmeas/ No. de ovos } \\
100 \\
\text { ovos }\end{array}$ & $\begin{array}{c}\text { No. de } \\
\text { parasitados } \\
\text { indivíduos/ } \\
\text { ovo }\end{array}$ & $\begin{array}{c}\text { Razão } \\
\text { sexual }\end{array}$ & $\begin{array}{c}\text { Viabilidade } \\
(\%)\end{array}$ \\
\hline 1 & $61,8 \pm 4,6$ b & $1,0 \pm 0,00$ a & $0,74 \pm 0,05$ a & $53,6 \pm 7,69$ a \\
3 & $90,2 \pm 6,6$ a & $1,0 \pm 0,00$ a & $0,44 \pm 0,19$ a & $53,4 \pm 19,99$ a \\
6 & $98,8 \pm 3,9$ a & $1,0 \pm 0,00$ a & $0,55 \pm 0,14$ a & $55,0 \pm 19,75$ a \\
9 & $99,8 \pm 2,1$ a & $1,0 \pm 0,00$ a & $0,63 \pm 0,16$ a & $58,6 \pm 23,96$ a \\
\hline
\end{tabular}

Médias seguidas da mesma letra, na coluna, não diferem significativamente, entre si, pelo teste de Tukey $(\mathrm{P} \leq 0,05)$. 
Tabela III. Parasitismo e razão sexual ( \pm EPM) de T. remus após diferentes períodos de ausência do hospedeiro $S$. frugiperda.

\begin{tabular}{ccc}
\hline $\begin{array}{c}\text { Idade das } \\
\text { fêmeas (dias) }\end{array}$ & $\begin{array}{c}\text { No. de ovos } \\
\text { parasitados }\end{array}$ & $\begin{array}{c}\text { Razão } \\
\text { sexual }\end{array}$ \\
\hline 1 & $97,0 \pm 2,52 \mathrm{a}$ & $0.59 \pm 0,08 \mathrm{~b}$ \\
2 & $92,9 \pm 4,41 \mathrm{a}$ & $0,74 \pm 0,04 \mathrm{a}$ \\
3 & $98,6 \pm 2,26 \mathrm{a}$ & $0,74 \pm 0,12 \mathrm{a}$ \\
4 & $100,0 \pm 0,80 \mathrm{a}$ & $0,78 \pm 0,02 \mathrm{a}$ \\
5 & $92,6 \pm 5,74 \mathrm{a}$ & $0,78 \pm 0,05 \mathrm{a}$ \\
6 & $98,9 \pm 6,35 \mathrm{a}$ & $0,84 \pm 0,02 \mathrm{a}$ \\
7 & $90,0 \pm 7,36 \mathrm{a}$ & $0,70 \pm 0,06 \mathrm{a}$ \\
8 & $92,7 \pm 7,72 \mathrm{a}$ & $0,68 \pm 0,09 \mathrm{a}$ \\
9 & $92,7 \pm 7,57 \mathrm{a}$ & $0,68 \pm 0,06 \mathrm{a}$ \\
10 & $61,1 \pm 3,32 \mathrm{~b}$ & $0,65 \pm 0,11 \mathrm{a}$ \\
\hline
\end{tabular}

Médias seguidas da mesma letra, na coluna, não diferem significativamente, entre si, pelo teste de Tukey $(\mathrm{P}<0,05)$ para a mesma característica.

Apesar de todos estes fatores, não detectaram-se mudanças na razão sexual quando as fêmeas encontravam-se sob condições de campo, mesmo em diferentes densidades (Tabela II). Mas, mesmo sob tais condições, o número de fêmeas manteve-se ainda maior do que o de machos.

Diversas pesquisas têm demonstrado que o número de parasitóides a ser liberado pode variar com a fenologia da planta, com a espécie e linhagem do parasitóide e com a dinâmica de postura do hospedeiro (Cardoso 2004; Pratissoli et al. 2005). Para T. remus, na cultura do milho, com plantas em estágio V4 a V6 (20 dias após a emergência), verificou-se que a liberação de 3 fêmeas/ 100 ovos de $S$. frugiperda é suficiente para que sejam obtidos níveis satisfatórios de parasitismo.

Verifica-se também que o número de fêmeas de $T$. remus por postura de $S$. frugiperda trata-se de fator importante a ser verificado no controle de qualidade das criações em laboratório e nas liberações massais, pois interfere em aspectos biológicos do parasitóide e quanto maiores forem os índices de parasitismo e o número de fêmeas de boa qualidade produzidas, a utilização de $T$. remus no controle de pragas pode ser amplamente expandida.

Efeito da ausência de hospedeiro. Constatou-se que mesmo depois de nove dias de ausência de hospedeiro não houve redução significativa nos índices de parasitismo por T. remus. Apenas no décimo dia pode-se observar queda no número médio de ovos parasitados (Tabela III).

O sucesso reprodutivo dos parasitóides depende da habilidade das fêmeas em ajustar a produção de ovos de acordo com a disponibilidade de hospedeiros (Vogt \& Nechols 1993; Hougardy et al. 2005) e, em alguns casos, a privação de hospedeiros pode ser utilizada para que se consigam melhores índices de fecundidade (Fleury \& Bouletreau 1993). No entanto, diante de tal situação, as fêmeas do inimigo natural podem acabar por reabsorver seus oócitos, ocasionando redução no parasitismo e na vida fértil (Hougardy et al. 2005).

A reabsorção de oócitos pode funcionar como uma forma de adaptação a períodos de escassez de hospedeiro ou condições ambientais desfavoráveis (Vogt \& Nechols 1993; Chabi-Olaye et al. 2001). Porém, outros fatores como a idade da fêmea, disponibilidade de alimento, fotoperíodo e temperatura podem influenciar diretamente a fecundidade (Vogt \& Nechols 1993)

Tal reabsorção geralmente encontra-se associada com a baixa proporção de oócitos maduros no momento de emergência da fêmea e também com o tempo que a mesma leva para encontrar o macho e efetivar a cópula (Van Alphen \& Jervis 1996; Farias \& Hopper 1997). Já as fêmeas de T. remus realizam a cópula imediatamente após a emergência, pois os machos auxiliam-nas a sair dos ovos do hospedeiro e realizam o acasalamento neste momento (Schwartz \& Gerling 1974; Bueno et al. 2008).

Em Trichogrammatidae, Pratissoli et al. (2004) constataram que existe um decréscimo no parasitismo ocasionado por Trichogramma galloi Zucchi 1988 (Hymenoptera, Trichogrammatidae), a partir de 48 h da vida do parasitóide e que quanto maior a ausência do hospedeiro a que são submetidas às fêmeas, maior é a taxa de reabsorção de seus ovos.

No entanto, algumas espécies de parasitóides, incluindo os pertencentes ao gênero Telenomus conseguem reter os ovos diante da ausência de hospedeiros e mantém suas taxas de parasitismo, mesmo depois de vários dias de privação, o que confere a estes inimigos naturais grande habilidade de estabelecimento em áreas de constante flutuação populacional da praga-alvo (Chabi-Olaye et al. 2001).

Quanto à razão sexual foi possível constatar que fêmeas com menos de $48 \mathrm{~h}$ de idade produzem menor quantidade de fêmeas na progênie (Tabela III). Houve diferença significativa entre as fêmeas com apenas $24 \mathrm{~h}$ de idade e as demais, porque T. remus tende a originar menor número de descendentes fêmeas ao ovipositarem no primeiro dia de vida (Welzen \& Waage 1987).

Conforme relataram Schwartz \& Gerling (1974), o número de machos na progênie de T. remus aumenta com a idade da fêmea, especialmente a partir do sexto dia de vida. Porém, neste ensaio não verificaram-se diferenças significativas neste aspecto (Tabela III).

Logo, pode-se concluir que $T$. remus adapta-se bem a ausência de hospedeiro, podendo permanecer em laboratório por alguns dias, caso não seja possível a liberação. Tal fato confere maiores possibilidades de uso desse inimigo natural nos programas de controle biológico, além do aprimoramento e otimização das criações massais.

Sugere-se ainda que novos estudos seja realizados a fim de verificar se fêmeas de T. remus que permanecem por alguns dias em laboratório atuariam de maneira semelhante àquelas recém-emergidas ao serem liberadas em campo. Ou mesmo, por quanto tempo fêmeas liberadas em campo permanecem na área alvo, mesmo na ausência do hospedeiro, o que poderia permitir liberações antecipadas.

\section{REFERÊNCIAS}

Bai, B. \& S. M. Smith. 1993. Effect of host availability on reproduction and survival of the parasitoid wasp Trichogramma minutum. Ecological Entomology 18: 279-286. 
Berti, J. \& R. Marcano. 1991. Effect of time of host absence on parasitism by Trichogramma pretiosum Riley (Hymenoptera: Trichogrammatidae). Boletín de Entomología Venezolana 6: $5-10$.

Beserra, E. B. \& J. R. P. Parra. 2005. Impact of the number of Spodoptera frugiperda egg layers on parasitism by Trichogramma atopovirilia. Scientia Agrícola 62: 190-193.

Bueno, R. C. O. F.; T. R. Carneiro; D. Pratissoli; A. F. Bueno \& O. A. Fernandes. 2008. Biology and thermal requirements of Telenomus remus reared on fall armyworm Spodoptera frugiperda eggs. Ciência Rural 38: 1-6.

Cardoso, A. M. 2004. Manejo de Spodoptera frugiperda (J. E. Smith, 1797) (Lepidoptera: Noctuidae) em milho, Zea mays L.: bases para avaliação populacional e controle biológico utilizando o parasitóide de ovos Trichogramma atopovirilia Otman \& Platner, 1983 (Hymenoptera: Trichogrammatidae). Tese (Doutorado), USP (FFCL), Ribeirão Preto, SP, 84 p.

Cave, R. D. 2000. Biology, ecology and use in pest management of Telenomus remus. Biocontrol News and Information 21: 2126.

Cave, R. D. \& N. M. Acosta. 1999. Telenomus remus Nixon: un parasitoide en el control biológico del gusano cogollero, Spodoptera frugiperda (Smith). Ceiba 40: 215-227.

Chabi-Olaye, A.; F. Schulthess; H. M. Poehling \& C. Borgemeister. 2001. Factors affecting the biology of Telenomus isi (Polaszek) (Hymenoptera: Scelionidae), an egg parasitoid of cereal stem borers in West Africa. Biological Control 21: 44-54.

Collins, M. D. \& A. F. G. Dixon. 1986. The effect of egg depletion on the foraging behavior of an aphid parasitoid. Journal of Applied Entomology 102: 342-352.

Cruz, I. 1995. A lagarta-do-cartucho na cultura do milho. Sete Lagoas (MG), EMBRAPA - CNPMS, (Circular Técnica Número 21), $45 \mathrm{p}$

Cruz, I. 2000. Métodos de criação de agentes entomófagos de Spodoptera frugiperda (J. E. Smith), p. 112-135. In: Bueno, V. H. P. (ed.). Controle biológico de pragas: Produção massal e Controle de qualidade. Lavras, Editora UFLA, 196 p.

Doutt, R. L. 1959. The biology of parasitic hymenoptera. Annual Review of Entomology 4: 161-182.

Farias, A. M. I. de \& K. R. Hopper. 1997. Responses of female Aphelinus asychis (Hymenoptera: Aphelinidae) and Aphidius matricarie (Hymenoptera: Aphidiidae) to host and plant-host odors. Biological Control 26: 989-994.

Fleury, F. \& M. Bouletreau. 1993. Effects of temporary host deprivation on the reproductive potencial of Trichogramma brassicae. Entomologia Experimentalis et Applicata 68: 203-210.

Garcia, M. A. 1991. Ecologia nutricional de parasitóides e predadores terrestres, p. 286-312. In: A. R. Panizzi \& J. R. P. Parra (eds.). Ecologia nutricional de insetos e suas implicações no manejo de pragas. São Paulo, Editora Manole, 359 p.

Gauld, I. \& B. Bolton. 1988. The Hymenoptera. London, Oxford University Press, 332 p.

Gerling, D. 1972. The developmental biology of Telenomus remus
Nixon (Hymenoptera: Scelionidae). Bulletin of Entomological Research 61: 385-388.

Hougardy, E.; T. M. Bezemer \& N. J. Mills. 2005. Effects of host deprivation and egg expenditure on the reproductive capacity of Mastrus ridibundus, an introduced parasitoid for the biological control of codling moth in California. Biological Control 33 96-106.

Oliveira, H. N.; J. C. Zanuncio; D. Pratissoli \& I. Cruz. 2000. Parasitism rate and viability of Trichogramma maxacalli (Hymenoptera: Trichogrammatidae) parasitoid of eucaliptus defoliator Euselasia apison (Lepidoptera: Riodinidae), on eggs of Anagasta kuehniella (Lepidoptera: Pyralidae). Forest Ecology Management 130: 16

Parra, J. R. P.; P. S. M. Botelho; B. S. Corrêa-Ferreira \& J. M. S. Bento. 2002. Controle biológico: Uma visão multidisciplinar, p. 125137. In: J. R. P. Parra; P. S. M. Botelho; B. S. Corrêa-Ferreira; J. M. S. Bento (eds). Controle biológico no Brasil: parasitóides e predadores. São Paulo, Editora Manole, 609 p.

Pratissoli, D.; H. N. de Oliveira; S. M. J. Vieira; R. C. Oliveira \& H. B. Zago. 2004. Efeito da disponibilidade de alimento nas características biológicas de Trichogramma galloi Zucchi (Hymenoptera: Trichogrammatidae). Revista Brasileira de Entomologia 48 101-104.

Pratissoli, D.; R. T. Thuler; G. S. Andrade; L. C. M. Zanotti \& A. F da Silva. 2005. Estimativa de Trichogramma pretiosum para controle de Tuta absoluta em tomateiro estaqueado. Pesquisa Agropecuária Brasileira 40: 715-718.

SAS Institute. 2004. SAS/STAT user's guide, release 9.1 ed. SAS Institute, Cary, NC.

Schwartz, A. \& D. Gerling. 1974. Adult biology of Telenomus remus (Hymenoptera: Scelionidae) under laboratory conditions. Entomophaga 19: 482-492.

Van Alphen, J. J. M. \& M. Jervis. 1996. Foraging behavior, p. 1-62. In: M. Jervis \& N. Kidd (eds). Insect natural enemies. London, UK, Chapman \& Hall, $491 \mathrm{p}$.

Vinson, S. B. 1998. The general host selection behavior of parasitoid Hymenoptera and a comparison of initial strategies utilized by larvaphagous and oophagous species. Biological Control 11: 7996.

Vogt, E. A. \& J. R. Nechols. 1993. The influence of host deprivation and host source on the reproductive biology and longevity of the squash bug egg parasitoid Gryon pennsylvanicum (Ashmead) (Hymenoptera: Scelionidae). Biological Control 3: 148-154.

Waage, J. K. 1982. Sib-mating and sex ratio strategies in scelionidae wasps. Ecological Entomology 7: 103-112.

Waage, J. K. \& M. P. Hassel. 1982. Parasitoids as biological control agents - a fundamental approach. Parasitology 84: 241-268.

Welzen, C. R. L. \& J. K. Waage. 1987. Adaptive responses to local mate competition by the parasitoid Telenomus remus. Behavioral Ecology and Sociobiology 21: 359-363.

Zera, A. J. \& L. G. Harshman. 2001. The physiology of life history trade-offs in animals. Annual Review of Ecological Systems 32: $95-126$.

Recebido em 13/12/2007; aceito em 22/05/2009 